\title{
High-Grade Atrioventricular Nodal Block: An Unusual Presentation of COVID-19 Infection
}

\author{
Sadeq Tabatabai Mosaad Abushabana Jasem Mohammed Alhashmi \\ Hosam Zaky \\ Cardiology Department, Dubai Hospital, Dubai Health Authority, Dubai, UAE
}

\section{Keywords}

Cardiology · Heart block · Atrioventricular nodal block . Infectious disease - Coronavirus disease 2019 infection

\begin{abstract}
While respiratory system disease, including pneumonia, remains the principal clinical presentation, several cardiovascular complications of coronavirus disease 2019 (COVID-19) including myocardial infarction, myocarditis, and tachyarrhythmias were reported. We present an unusual case of fatal high-grade atrioventricular nodal block in a young adult male with COVID-19 infection in the occurrence of normal coronary arteries. Marked inflammatory response and myocarditis are the most likely contributing factors to electrical conduction system disturbance. It is appropriate for the clinicians to be aware of the fact that COVID-19 can atypically present as bradyarrhythmia and conduction abnormalities.

(C) 2021 The Author(s)

Published by S. Karger AG, Basel
\end{abstract}

\section{Introduction}

Although respiratory tract disease, including pneumonia, remains the main clinical presentation, several cardiovascular complications of coronavirus disease
2019 (COVID-19) including myocardial infarction, myocarditis, cardiomyopathy, stroke, and venous thromboembolic disease including pulmonary embolism have been documented and reported during the current pandemic [1]. Additionally, arrhythmias are another cardiovascular manifestation commonly described in patients with COVID-19 infection, specifically cardiac tachyarrhythmias in the existence of underlying myocarditis, acute coronary syndrome, hypoxia, electrolyte disturbances caused by renal impairment, or a side effect of drug therapy [2]. Cardiac involvement in patients with COVID-19 may be reflected by dynamic electrocardiographic (ECG) changes which could be the hallmark of cardiac injury and usually signifies a critical status in infected patients [3].

Furthermore, congenital channelopathy, like Brugada syndrome leading to syncope with loss of consciousness, can be unmasked by severe acute respiratory syndrome coronavirus 2 (SARS-CoV-2)-induced fever which requires implantable cardioverter-defibrillator insertion [4]. The episodes of bradycardia and atrioventricular (AV) block are usually been reported in the setting of inferior wall myocardial infarction, and we describe an unusual case of fatal high-grade AV nodal block in a young adult male with COVID-19 infection.
C 2021 The Author(s)

Published by S. Karger AG, Basel

This is an Open Access article licensed under the Creative Commons Attribution-NonCommercial-4.0 International License (CC BY-NC) (http://www.karger.com/Services/OpenAccessLicense), applicable to the online version of the article only. Usage and distribution for commercial purposes requires written permission.
Correspondence to:

Sadeq Tabatabai, drsadeq@yahoo.com 
Fig. 1. ECG finding at presentation. $\mathrm{P}$ waves marked with arrows. a ECG from the cardiac monitor of the ambulance showing nonconducting $\mathrm{P}$ wave with long significant pause suggestive of significant $\mathrm{AV}$ nodal block. b ECG on arrival to ED showing second-degree, type II AV nodal block. Average ventricular rate was $39 \mathrm{bpm}$, and atrial rate was around $150 \mathrm{bpm}$ suggestive of P:QRS predominantly 4:1 conduction rate. c ECG after starting dopamine infusion showing improvement in the ventricular rate response.

Fig. 2. a Angiogram of the left coronary artery system showing normal coronaries. b Angiogram of the right coronary artery showing normal coronary flow.
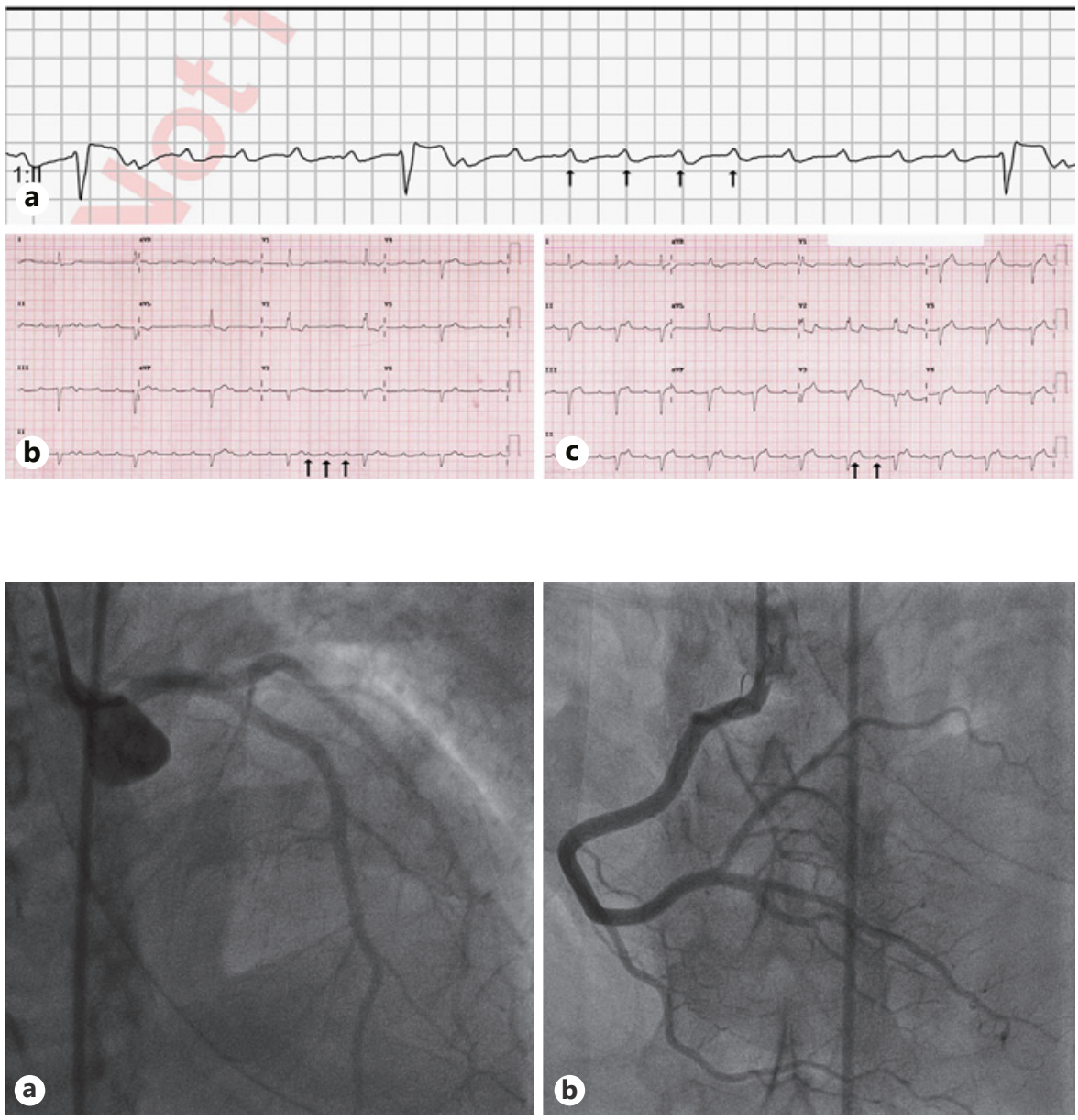

\section{Case Report}

This is an otherwise healthy young 35-year-old male who presented to the emergency department (ED) at Dubai hospital with a history of recurrent giddiness and presyncope of 2-day duration. He gave a history of fever as well. He denied any chest pain. He had no significant past medical history, and he was not on any regular medication.

On arrival to $\mathrm{ED}$, he was conscious but drowsy. Initial vital signs showed a blood pressure (BP) of 74/48 $\mathrm{mm} \mathrm{Hg}$ (BP location: left arm, patient position: lying), pulse 37 beats per minute (bpm), Temp $38.8^{\circ} \mathrm{C}\left(101.8^{\circ} \mathrm{F}\right)$ (tympanic), respiratory rate 20 breaths per minute, oxygen saturation $\left(\mathrm{SpO}_{2}\right) 88 \%$, and $\mathrm{BMI} 25.30 \mathrm{~kg} / \mathrm{m}^{2}$. His clinical examination at time of presentation was unremarkable apart from drowsiness and hypotension, and he was febrile.

ECG strip from the cardiac monitor (ambulance) showed nonconducting $\mathrm{P}$ wave with long significant pause approximately 4,200 ms suggestive of significant AV nodal block. Initial resting ECG showed evidence of second-degree, type II AV nodal block (Fig. 1).

He made a good clinical response to the initial resuscitation measures with oxygen supplement and intravenous fluid and dopamine infusion, and hence insertion of a temporarily pacemaker was not needed. His BP improved to $123 / 68 \mathrm{~mm} \mathrm{Hg}$, pulse $86 \mathrm{bpm}$,
Temp $37.7^{\circ} \mathrm{C}\left(99.9^{\circ} \mathrm{F}\right)$, and $\mathrm{SpO}_{2} 96 \%$. Glasgow Coma Scale Score was 15.

Resting ECG while on dopamine infusion showed second-degree, type II AV nodal block with ST changes mainly in the inferior leads what was thought to be inferior wall ischemia (Fig. 1c). Ventricular rate improved up to $66 \mathrm{bpm}$. Initial bedside echocardiogram showed good left ventricular systolic function and left ventricular ejection fraction (eyeballing estimation) about 55\%, and there were no significant regional wall motion abnormalities. While waiting for transfer to the inpatient unit, his SARS-CoV-2 reverse transcriptase polymerase chain reaction assay of nasopharyngeal swab was found to be positive.

Initial laboratory tests showed peak troponin $14,797 \mathrm{ng} / \mathrm{L}$, serum ferritin 10,098 $\mathrm{ng} / \mathrm{mL}$, D-dimer test $10.24 \mu \mathrm{g} / \mathrm{mL}$, peak creatine kinase $\mathrm{MB}$ isoenzyme mass $205.0 \mathrm{ng} / \mathrm{mL}$, creatine phosphokinase $1,313 \mathrm{U} / \mathrm{L}, \mathrm{N}$-terminal pro-brain natriuretic peptide 22,272 $\mathrm{pg} / \mathrm{mL}$, C-reactive protein $41.9 \mathrm{mg} / \mathrm{L}$, procalcitonin $0.42 \mathrm{ng} / \mathrm{mL}$, and interleukin-6 $180.0 \mathrm{pg} / \mathrm{mL}$. At this stage, he was started on dual antiplatelet therapy in the form of aspirin and clopidogrel and a therapeutic dose of enoxaparin and tirofiban infusion along with initial normal saline and dopamine infusion. He was kept in high dependency isolation unit with a cardiac monitor, and he was started on favipiravir according to the protocol of the COVID team. 
Fig. 3. a Chest X-ray on arrival showing prominent bronchovascular markings. b Chest X-ray after insertion of endotracheal tube and mechanical ventilation showing worsening of a bilateral diffuse lung infiltrates, right lung more than the left.
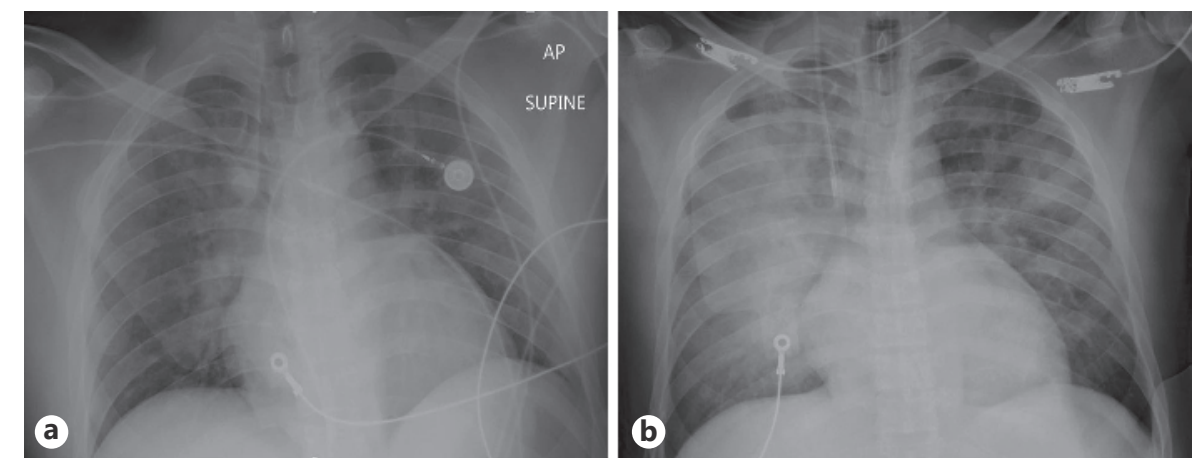

He remained relatively stable until about $10 \mathrm{~h}$ after admission, when he suddenly deteriorated and became distressed, tachycardic, and severely dyspneic with clinical evidence of shock requiring intratracheal intubation and mechanically ventilated with high doses of vasopressors to maintain favorable hemodynamics. It was decided to take him for urgent coronary angiogram which showed normal coronary arteries (Fig. 2). Chest X-ray showed worsening of bilateral diffuse lung infiltrates, right lung more than the left (Fig. 3).

At approximately $15 \mathrm{~h}$ after admission, he suddenly developed bradycardia and asystole. Cardiopulmonary resuscitation was immediately carried out as per advanced cardiac life support protocol, but return of spontaneous circulation could not be achieved, and unfortunately he died ultimately.

\section{Discussion}

Arrhythmia in general is one of the potential cardiac sequelae that may result from COVID-19 infection. Even though it is relatively uncommon, there are growing pieces of evidence showing that cardiac bradyarrhythmias are also one of the major complications of COVID-19. Perhaps, it is not yet well known whether a definitive primary mechanism for development of bradyarrhythmia is a result of myocardial and/or conduction system damage, or the virus has a direct arrhythmogenic effect due to likeness for the receptors in the myocardial conduction system. As myocarditis has been reported in many patients with COVID-19, it is acceptable that myocardial inflammation and injury may affect the conduction system resulting in high-grade AV block [2].

Studies have suggested that myocardial injury is usual especially in critically ill COVID-19-infected patients through different mechanisms mostly due to direct damage of cardiomyocytes and systemic inflammation [5]. Inflammatory cells and SARS-CoV-2 virus have been identified in the myocardium in autopsy studies, suggesting direct invasion of the heart in some cases [6].
Nevertheless, local inflammation of the myocardium due to effects of the inflammatory response of the cardiac tissue directed to the SARS-CoV-2 virus leading to isolated conduction system disturbances is another thinkable mechanism for high-grade AV block in infected patients as it was reported with other infectious pathogens [7]. Arrhythmias can often be the first presenting feature of myocarditis. Acute and chronic myocarditis is considered to be among the most important causes of advanced AV block in young and middle-aged patients [8].

Almost all sites of the cardiac conduction system can be affected by COVID-19 infection. Sinus node dysfunction, including sick sinus syndrome and sinus arrest (causing long pause and asystole requiring pacemaker insertion), and different types of AV node block, including high-degree AV and complete heart block, have been reported during the current pandemic $[9,10]$.

Accordingly, in our case study, concomitant high-degree AV nodal block and COVID-19 is almost certainly due to acute inflammation of the myocardium and cardiac conduction system, evident by raising of the cardiac troponin and inflammatory markers, leading to myocarditis and interruption of the myocardial electrical conduction. The other possible trigger is acute myocardial ischemia, but that is unlikely due to the absence of anginal chest pain and normal coronary artery flow.

The management of bradycardia is still according to the advanced cardiac life support guidelines for symptomatic patients with initial pharmacological intervention. Although pacemaker insertion is indicated for symptomatic hemodynamically unstable patients, patients with heart block associated with COVID-19 infection might have poor outcome and prognosis despite pacing support and in-hospital and 3-month mortality rate estimated up to $57 \%$ and $70 \%$, respectively, in some small reported case series [10]. 
In patients presenting with what seems to be a typical cardiac disorder, COVID-19 infection should be always in the background of the differential during the present pandemic, even in the absence of the classical symptoms, especially when the presenting cardiac condition is not matching with patient's clinical profile. We believe it is applicable for the clinicians to be aware of such worrisome potential effect of SARS-CoV-2 infection, and thus our aim in presenting this case report is to create alertness of a possible atypical effect of this virus on the cardiac conduction system.

\section{Conclusion}

The involvement of the conduction system is favorably affected by myocardial inflammatory response and myocarditis caused by the virus. It is appropriate for the clinicians to be aware of the fact that COVID-19 can uncharacteristically present as bradyarrhythmia and conduction disturbances.

\section{Statement of Ethics}

This case report complies with the guidelines for human studies and was conducted ethically in accordance with the World Medical Association Declaration of Helsinki. Ethical approval was not required for this case report in accordance with the Dubai Scientific Research Ethics Committee policies. Written informed consent for publication of the case report and any accompanied images was obtained from the patient's next of kin.

\section{Conflict of Interest Statement}

The authors declared no potential conflicts of interest with respect to the research, authorship, and/or publication of this article.

\section{Funding Sources}

The authors received no financial support for the research, authorship, and/or publication of this article.

\section{Author Contributions}

All the authors contributed equally in writing the manuscript. All authors read and approved the final manuscript.

\section{Data Availability Statement}

All data generated or analyzed during this study are included in this article. Further enquiries can be directed to the corresponding author.

\section{References}

1 Fried JA, Ramasubbu K, Bhatt R, Topkara VK, Clerkin KJ, Horn E, et al. The variety of cardiovascular presentations of COVID-19. Circulation. 2020;141(23):1930-6.

2 Driggin E, Madhavan MV, Bikdeli B, Chuich T, Laracy J, Biondi-Zoccai G, et al. Cardiovascular considerations for patients, health care workers, and health systems during the $\mathrm{CO}$ VID-19 pandemic. J Am Coll Cardiol. 2020; 75(18):2352-71.

3 He J, Wu B, Chen Y, Tang J, Liu Q, Zhou S, et al. Characteristic electrocardiographic manifestations in patients with COVID-19. Can J Cardiol. 2020;36(6):966-e4.
4 Pasquetto G, Conti GB, Susana A, Leone LA, Bertaglia E. Syncope, Brugada syndrome, and COVID-19 lung disease. J Arrhythm. 2020; 36(4):768-70.

5 Babapoor-Farrokhran S, Gill D, Walker J, Rasekhi RT, Bozorgnia B, Amanullah A. Myocardial injury and COVID-19: possible mechanisms. Life Sci. 2020;253:117723.

6 Bansal M. Cardiovascular disease and COVID-19. Diabetes Metab Syndr. 2020;14(3): $247-50$.

7 Forrester JD, Mead P. Third-degree heart block associated with lyme carditis: review of published cases. Clin Infect Dis. 2014;59(7): 996-1000.
8 Barra SN, Providência R, Paiva L, Nascimento J, Marques AL. A review on advanced atrioventricular block in young or middle-aged adults. Pacing Clin Electrophysiol. 2012; 35(11):1395.

9 Gupta MD, Qamar A, Mp G, Safal S, Batra V, Basia D, et al. Bradyarrhythmias in patients with COVID-19: a case series. Indian Pacing Electrophysiol J. 2020;20(5):211-2.

10 Chinitz JS, Goyal R, Harding M, Veseli G, Gruberg L, Jadonath R, et al. Bradyarrhythmias in patients with COVID-19: marker of poor prognosis? Pacing Clin Electrophysiol. 2020; 43(10):1199-204. 